How to cite this article:

Ramli, R., Abdul Rahman, R., \& Rohim, N. (2019). A hybrid ant colony optimization algorithm for solving a highly constrained nurse rostering problem. Journal of Information and Communication Technology, 18(3), 305-326.

\title{
A HYBRID ANT COLONY OPTIMIZATION ALGORITHM FOR SOLVING A HIGHLY CONSTRAINED NURSE ROSTERING PROBLEM
}

\author{
Razamin Ramli, Rosshairy Abd Rahman \& Nurdalila Rohim \\ School of Quantitative Sciences, \\ Universiti Utara Malaysia, Malaysia
}

razamin@uum.edu.my; shairy@uum.edu.my;nurdalilarohim@gmail.com

\begin{abstract}
Distribution of work shifts and off days for nurses in a duty roster is a crucial task. Much effort is spent trying to produce workable and quality rosters for nurses in hospital wards. However, there are cases, such as mandatory working days per week and balanced distribution of shift types that cannot be achieved in manually generated rosters, which are still being practised today. Hence, this study focused on solving these issues arising in Nurse Rostering Problems (NRPs) strategizing on a hybrid of Ant Colony Optimization (ACO) algorithm with a hill climbing technique. The hybridization with hill climbing was aimed at fine-tuning the initial solution or roster generated by the ACO algorithm to achieve better rosters. The hybrid model was developed with the goal of satisfying hard constraints, while minimizing violations of soft constraints in such a way that fulfilled hospital rules and nurses' preferences. The real data used for the highly constrained NRP was obtained from a large Malaysian hospital. There were specifically, three main phases involved in developing the hybrid model: generating an initial roster; updating the roster through
\end{abstract}


the ACO algorithm, and implementing the hill climbing to further search for a refined solution. The results showed that with a larger value of pheromone, the chances of obtaining a good solution was found with only small penalty values. This study has proven that the hybrid ACO is able to solve NRPs with good potential solutions that satisfied all four important criteria: coverage, quality, flexibility, and cost. Subsequently, the hybrid model is also beneficial to the hospital's management as nurses can be scheduled with a balanced distribution of shifts, which in turn fulfilled their preferences.

Keywords: Ant colony optimization, metaheuristic technique, hybridization strategy, hill climbing, nurse rostering problem

\section{INTRODUCTION}

In an organization like hospitals, the staffing and scheduling processes of nurses are important designs or components that may affect organizational performance (Joseph, 2018). The reason is to ensure that there is sufficient number of nurses on duty and that all nurses are treated fairly in the distribution of work shifts. Typically, a duty roster for nurses needs to satisfy the work regulations and demands of each nurse, and at the same time satisfy the preferences of the nurses. The rules involved in constructing a roster contribute to the problem of nurse rostering, which is a subclass of the scheduling problem that is challenging to be solved (Joseph, 2018; Kundu \& Acharyya, 2008). This NRP involves allocating workload to nurses subject to several restrictions or constraints. Normally, a nurse rostering expert has to spend much of his/her time making and updating rosters, manually. The aim is to produce high quality rosters, wherein the well-being of nurses is taken care of without dismissing the concerns of employers (Joseph, 2018; Özcan, 2005; Royal College of Physicians, 2018). Hospital operations of 24 hours a day and 7 days a week can affect the changes in nurses' daily lives, which could also lead to health problems (Joseph, 2018; Royal College of Physicians, 2018). Thus, in order to ensure the continuity of services, shifts in the working environment is required and this has been practised in most hospitals. In relation to that, the development of a high-quality nurse roster that is fair and reasonable to each nurse is a must. As a result, studies on the development or modeling of the best roster in terms of quality are still relevant and ongoing. 
Consequently, a number of solutions or rostering models have been recommended to solve NRPs through several methodologies, such as the Tabu Search (Dowsland \& Thompson, 2000), Memetic Algorithm (Ramli, Mustafa, \& Khader, 2004), Goal Programming (Azaiez \& Al Sharif, 2005), Integer Programming (Trilling, Giunet, \& Magny, 2006), Genetic Algorithm (Moz \& Pato, 2007), Ant Colony Optimization (ACO) (Gutjahr \& Rauner, 2007), Fuzzy Logic (Eskandari \& Ziarati, 2008), Variable Neighborhood Search (Croce \& Salassa, 2014), Hybrid Artificial Bee Colony (Awadallah, Bolaji, \& Al-Betar, 2015), Hybrid Harmony Search (Awadallah, Al-Betar, Khader, Bolaji, \& Alkoffash, 2017), Evolutionary Algorithm (Ramli \& Lim, 2017) and Simulated Annealing (Liu, Liu, Zhu, Shen, \& Dong, 2018). Among these studies, the ACO metaheuristic methodology is best suited to a dynamic problem environment such as the NRP and it provides positive feedback that leads to good feasible solutions. In fact, Gutjahr and Rauner (2007) revealed that the ACO offers significant results, when compared to other algorithms in solving NRPs. However, the ACO technique could be further improvised to provide new potential solution models as motivated by Awadallah et al. (2017) with their Harmony Search that integrates the Hill Climbing $(\mathrm{CH})$ technique to produce better solutions for the NRP. This is in line with current research trends in exploiting the hybridization of two or more techniques to provide high quality solutions, which has been evidenced in Awadallah et al. (2015). Hence, this study on NRP aims to propose a hybrid ACO, where the Hill Climbing technique is integrated so as to provide high quality rosters for the nursing workforce, specifically in terms of coverage, quality, flexibility and cost.

Subsequently, the rest of this paper is organized in the following sections: some reviews on related NRPs, methodology of a proposed nurse rostering model, followed by results and discussion. The final section concludes the paper along with some recommendations for future research.

\section{RELATED REVIEWS ON NURSE ROSTERING}

The problem of nurse rostering is also known as an NP-Complete problem (Winstanley, 2004; V'aclav'ik, S ucha, \& Hanz'alek², 2016). This condition is due to the complexity of the NRP, which is contributed by the variations in nurses' qualifications, contradictions among the large number of lawful management and staff requirements, fulfillment of general and specific working patterns, and a wide variety of other constraints. These constraints are typically categorized into two groups; hard and soft constraints (Özcan, 2005; $\mathrm{V}^{\prime}$ aclav' $1 \mathrm{k}$ et al., 2016). The complexity of the NRP also adds to the difficulty 
in developing automated scheduling systems that can produce quality rosters in a short time (Chiaramonte, 2008).

Many approaches have been proposed by various researchers to solve NRPs. The three main approaches frequently used in solving the problems are mathematical programming (MP), heuristics, and artificial intelligence (AI) (Cheang, Li, \& Rodrigues, 2003). These approaches can be further classified into two main categories, i.e., optimization and heuristic approaches. The optimization approach is basically based on MP techniques, while the heuristic approach usually employs constructive heuristics and/or metaheuristic techniques. A review by Rohim (2013) revealed that studies concerning nurse rostering are dominated by metaheuristic techniques. Although many researches have probed into this particular area, only a few methods have been tested using real world data (Burke, Curtois, Post, Qu, \& Veltman, 2005).

A metaheuristic technique can be defined as an iterative generation procedure that intelligently combines concepts of exploring and exploiting search space, where learning strategies are used to efficiently seek near-optimal solutions (Osman \& Laporte, 1996). Various metaheuristic techniques have since been utilized in NRPs, such as the Scatter Search (Burke, Curtois, Qu, \& Berghe, 2007), Tabu Search (Dowsland, 1998; Dowsland \& Thompson, 2000; Bester, Nieuwoudt, \& Vuuren, 2007; Baumelt et al., 2007), Genetic Algorithm (Aickelin \& Dowsload, 2000; Aickelin \& Dowsland, 2003; Moz \& Pato, 2007), agent-based scheduling (Aknine, Pinson, \& Shakun, 2004; Haspeslagh, Causmaecker, \& Berghe, 2007; Chiaramonte \& Chiaramonte, 2008), Memetic Algorithm (Özcan, 2005; Gonsalves \& KoheiKuwata, 2015), Variable Neighborhood Search (Burke et al., 2008; Croce \& Salassa, 2014), Simulated Annealing (Kundu, Mahato, Mahanty, \& Arharyya, 2008; Liu et al., 2018), Hybrid Harmony Search (Awadallah, et al., 2017), Hybrid Artificial Bee Colony (Awadallah, Bolaji, \& Al-Betar, 2015), Great Deluge (Arajy, Abdullah, \& Kifah, 2017) and ACO (Gutjahr \& Rauner, 2007; Wu, Lin \& Chen, 2013; Pooja \& Rupali, 2014). Although metaheuristic techniques have been experimented widely in NRPs, each technique seems to provide solutions to certain specific problems. Therefore, the solution for each technique is somewhat different and therefore it is not suitable to make comparisons.

Many metaheuristics are the results of hybridization of two or more techniques. For example, the hybridization of ACO and Hill Climbing were exploited in bandwidth minimization problems by Lim et al. (2006). This approach had shown relatively good performance. Meanwhile, Hayakawa et al. (2011) 
employed an ACO-Hill Climbing hybrid for a Constraint Satisfaction Problem (CSP). In their studies, the search inefficiency in the ACO was improved by the Hill Climbing procedure. Hill Climbing is a local search technique that starts with an arbitrary solution to a problem, then attempts to find a better solution by making an incremental change to the solution. If the change produces a better solution, another incremental change is made to the new solution until no further improvements can be made. This technique was applied to a large-scale and hard binary CSP instances in phase transition regions, whose experimental simulations demonstrated the efficiency of their hybrid technique. Other studies that exploited the benefits of ACO-Hill Climbing hybrid were Yousefikhoshbakht (2013) for the Travelling Salesman Problem (TSP) and Oonsrikaw and Thammano (2018) for a vehicle routing problem. They demonstrated that the newly proposed model performed better than the existing model. Another current study with ACO-Hill Climbing hybrid was developed for a TSP by Ratanavilisagul and Pasaya (2018) with good solutions as well.

It is acknowledged that, the ACO possesses a beneficial ability of giving feedback in its procedure such that the positive ones would lead to a quick discovery of good solutions and yet able to avoid premature convergence (Abreu, Ajmal, Kokkinogenis, \& Bozorg, 2001). However, only a limited number of studies have taken advantage of this technique in solving the NRPs. One such effort was by Gujarth and Rauner (2007), where the ACO was exploited to retain the memory of the entire colony instead of only one generation, which was less affected by poor initial solutions due to the combination of colony memory and random path selection. Moreover, Gujarth and Rauner (2007) found that Simulated Annealing and Genetic Algorithm were rather ineffective due to the complex form of the nurse rostering constraints. On the other hand, Awadallah et al. (2017) employed a metaheuristic technique whereby a Harmony Search was integrated with an established local search technique, i.e., the Hill Climbing for a highly constrained NRP to achieve good solutions. In this situation, many hard and soft constraints including nurse preferences need to be fulfilled. In fact, Hill Climbing was also integrated with Cuckoo Search Algorithm for global optimization problems (Shehab, Khader, \& Laouchedi, 2018) and with Evolutionary Algorithms for classification problems (Ibrahim, Shamsuddin, \& Qasem, 2015). In almost all cases, the hybridization of two or more techniques generated better solutions in any problem domain. Hence, these studies have ignited a keen interest to explore the ability of ACO in a hybridized manner in our study. 


\section{METHODOLOGY}

The ACO technique was deemed appropriate in this study, to be utilized in solving a highly constrained NRP as recommended by Gujarth and Rauner (2007), which was further improved by an effective local search technique, i.e., the Hill Climbing as suggested by several reviewed studies (Lim et al., 2006; Hayakawa et al., 2011; Awadallah et al., 2017; Oonsrikaw \& Thammano, 2018). Our proposed model, in particular, was inspired by the proven ACO as an effective technique for highly constrained problems (Gujarth \& Rauner, 2007). Thus, the development of the proposed hybrid ACO model for a special and highly constrained NRP is described in this methodology section. The hybrid model was designed with the ACO algorithm as the main strategy, whereby the Hill Climbing was later introduced to fine-tune prior nurse rostering solutions and then to recommend one as the final best-so-far solution. The important function of the Hill Climbing was to explore the ACO solution space and search for possibilities of arriving at a better solution based on the constraints in the NRP. Before the development of the hybrid model, the problem of nurse rostering environment which includes all available data and constraints tied to this specific NRP are described in the following subsections.

\section{Data and Nurse Rostering Environment}

Constructing an efficient schedule or roster that can provide fairness in the distribution of work shifts in a particular department or ward is crucial in healthcare services. In this study, a Special Care Nursery (SCN) ward in a large Malaysian hospital was selected to support the development of the proposed model, where a total of 39 staff nurses (SNs) constituted the nursing workforce. This ward is dedicated to nursing babies and toddlers and operates on a 24-hour service. The shifts rotate through three periods of time in a day, which are the morning shift (07:00-14:00), evening shift (14:00PM-21:00PM), and night shift (21:00-07:00). Rest days are off days, which are classified as weekly off day, public off day, the entitled annual leave and the off day due to working in three consecutive night shifts. Table 1 summarizes the type of work shifts along with its corresponding duration, off days and designated symbols. For instance, the symbol M represents the morning shift, while $\mathrm{E}$ and $\mathrm{N}$ represent evening and night shifts, respectively. This important information serves as data for the NRP including all specific constraints as discussed in the following sub-sections. All of the information and data were collected based on regulations imposed by the SCN ward and also from interviews with the head nurse and nurses (Ramli \& Lim, 2017). 
Table 1

Type of work shifts and off days

\begin{tabular}{lcc}
\hline & Duration & Symbol \\
\hline Morning & $07: 00-14: 00$ & $\mathrm{M}$ \\
Evening & $14: 00-21: 00$ & $\mathrm{E}$ \\
Night & $21: 00-07: 00$ & $\mathrm{~N}$ \\
Weekly off day & Nil & WO \\
Public off day & Nil & PO \\
Annual leave & Nil & AO \\
Night off day & Nil & NO \\
\hline
\end{tabular}

\section{Consideration of Constraints}

Constraints or requirements can be categorized into hard and soft constraints. Hard constraints are constraints that must be satisfied in order to obtain a feasible solution. Meanwhile, soft constraints are constraints that are good to fulfill but would not affect the core quality of the roster if violated. Hence, they are aimed to be satisfied as much as possible. The specific hard and soft constraints are listed in this section, where the meaning of each type of constraint is stated. The mandatory working day constraint refers to the number of working days that are required for a nurse to work in a week with one off day entitlement in each week. In addition, the work stretch constraint refers to the number of consecutive working days allowed with a minimum and maximum number of days. As a whole, the covering constraint ensures that a minimum number of nurses are assigned for each work shift and in each skill level in a day. The work requirement constraint emphasizes that the number of work shifts allowed is only one shift in a day (24-hour) regardless of the type of shift. Another constraint is the pre-assigned constraint that controls the assignment of night shifts according to turns or rotations, whereby two off days are automatically given after the third night shift. Finally, the ordering constraint ensures that the assignment of all types of shifts must follow a forward clockwise direction rule or the circadian rhythm to avoid negative effects on the disturbances of the body clock (Fumiko, 2014).

Subsequently, there are two types of soft constraints which are: days off arrangement constraint and shift arrangement constraint. The former refers to the arrangement of days off that are strongly and commonly preferred by nurses, while the latter is on the combinations of morning and evening shifts such that they are equal or almost equal in number. The penalty value for each 
violation of the soft constraint differs based on the priority of the constraint.

\section{Hard Constraints}

1. Nurses are to work six (6) days a week with the entitlement of one day off in each week (Mandatory Working Days Constraint).

2. Consecutive working days must not be less than two (2) days, but must not exceed six (6) days. Thus, split off days or single working day is disallowed (Work Stretch Constraint).

3. At least three (3) nurses must be allocated for each shift, consisting of each skill level in each day (Covering Constraint).

4. A nurse is only allowed to work one (1) shift in a 24-hour day (Work Requirement Constraint).

5. N shifts are assigned in blocks of three (3) shifts according to turns and rotations (Pre-assigned Constraint).

6. Two (2) days off is required to follow the third N shift of the block (Preassigned Constraint).

7. In any adjacent work shifts for each nurse, formation of night $(\mathrm{N})$ morning (M) shifts are prohibited (Ordering Constraint).

8. The assignment of shifts should follow the forward clockwise direction rule or circadian rhythm, i.e. $\mathrm{M} \rightarrow \mathrm{E} \rightarrow \mathrm{N}$ (Ordering Constraint).

\section{Soft Constraints}

1. Off days are strongly preferred to be consecutive in the arrangement (Days off Arrangement Constraint).

2. The combinations of $\mathrm{M}$ and $\mathrm{E}$ be $4 \mathrm{Ms} 2 \mathrm{Es}, 3 \mathrm{Ms} 3 \mathrm{Es}$ or $2 \mathrm{Ms} 4 \mathrm{Es}$ are preferred in a stretch of six (6) working days with no $\mathrm{N}$ shift. In this case, the minimum number of $\mathrm{M}$ is 2 , while the maximum number of $M$ is 4 , and vice versa with $E$. Thus, $2 \leq M \leq 4$ and $2 \leq E \leq 4$ (Shift Arrangement Constraint).

3. Similarly, for a 5-day work stretch, the preferred combinations of $\mathrm{M}$ and $\mathrm{E}$ are $2 \leq \mathrm{M} \leq 3$ and $2 \leq \mathrm{E} \leq 3$ (Shift Arrangement Constraint).

4. It is also preferred that for a 4-day stretch, the combination is $2 \mathrm{Ms}$ and 2Es (Shift Arrangement Constraint).

5. For 2-day and 3-day stretches, the following is preferred: 2Ms or 2Es and $3 \mathrm{Ms}$ or $3 \mathrm{Es}$, respectively (Shift Arrangement Constraint).

\section{Development of a Hybrid Ant Colony Optimization Model}

We proposed a hybrid ACO model to generate high quality rosters that are able to take into consideration all related data and constraints. The model is 
designed in such a way that all work shift slots for each nurse in each day are allocated, fulfilling the hard constraints and minimizing violations of any soft constraints. For each good roster, the pheromone value is increased. However, in terms of the objective function of the model, it is computed to minimize penalties when soft constraints are violated. The objective function is to:

$$
\operatorname{Min} Z=\sum_{i=1}^{I} \sum_{j=1}^{J} A_{i j o} \varepsilon_{j o}+\sum_{j=1}^{J} \rho_{j p} C_{p t j d}+\sum_{j=1}^{J} \varphi_{j s} D_{s t j d}+\sum_{j=1}^{J} \psi_{j l} E_{j v}
$$

Equation (1) is to compute the minimization value of four types of penalty i.e., penalty values assigned to type of nurse, shift arrangement, working stretch and non-working stretch (or days off arrangement). The nomenclature for each symbol is defined as follows:
$Z$ : Total value of penalties
$T$ : Total no. of days on shift timetable
$J$ : Total number of nurses in the ward
$I$ : Indicating day $I, i=1,2, \ldots, I$
$P$ : Shift arrangement
$S:$ Stretch of working days
$O$ : Shift type

- The parameters are as follows:

$\varepsilon_{j o}$ penalty nurse $j=1, \ldots, 39$ for nurse type $o$

$\rho_{j p}$ penalty nurse $j$ for shift arrangement $p$

$\varphi_{j s}$ penalty nurse $j$ for working stretch $s$

$\psi j l$ penalty nurse $j$ for non-working stretch $l$

- Decision variable
$\mathrm{A}_{i j o}$ assignment of nurse type $o(0=$ not assigned or $1=$ assigned $)$
$\mathrm{C}_{p t j}$ number of shift arrangement for nurse $j$
$\mathrm{D}_{s t j}$ number of working stretch for nurse $j$
$\mathrm{E}_{j v}$ number of non-working stretch $v$

The procedure of the hybrid ACO algorithm are illustrated in Figure 1 and is adapted from Zhang et al. (2006) for the job scheduling problem. The three main phases involved in the development of nurse rosters are initial generation of roster, which is Step 1, followed by updating the roster using the ACO algorithms, as explained in Steps 2 and 3, and finally, implementing the Hill Climbing technique to refine the solution, as discussed in Step 4. Steps 5 to 7 
depict the evaluation process of calculating the penalty values obtained from the ACO and hill climbing until the best solution is found. The details for each step are discussed as follows.

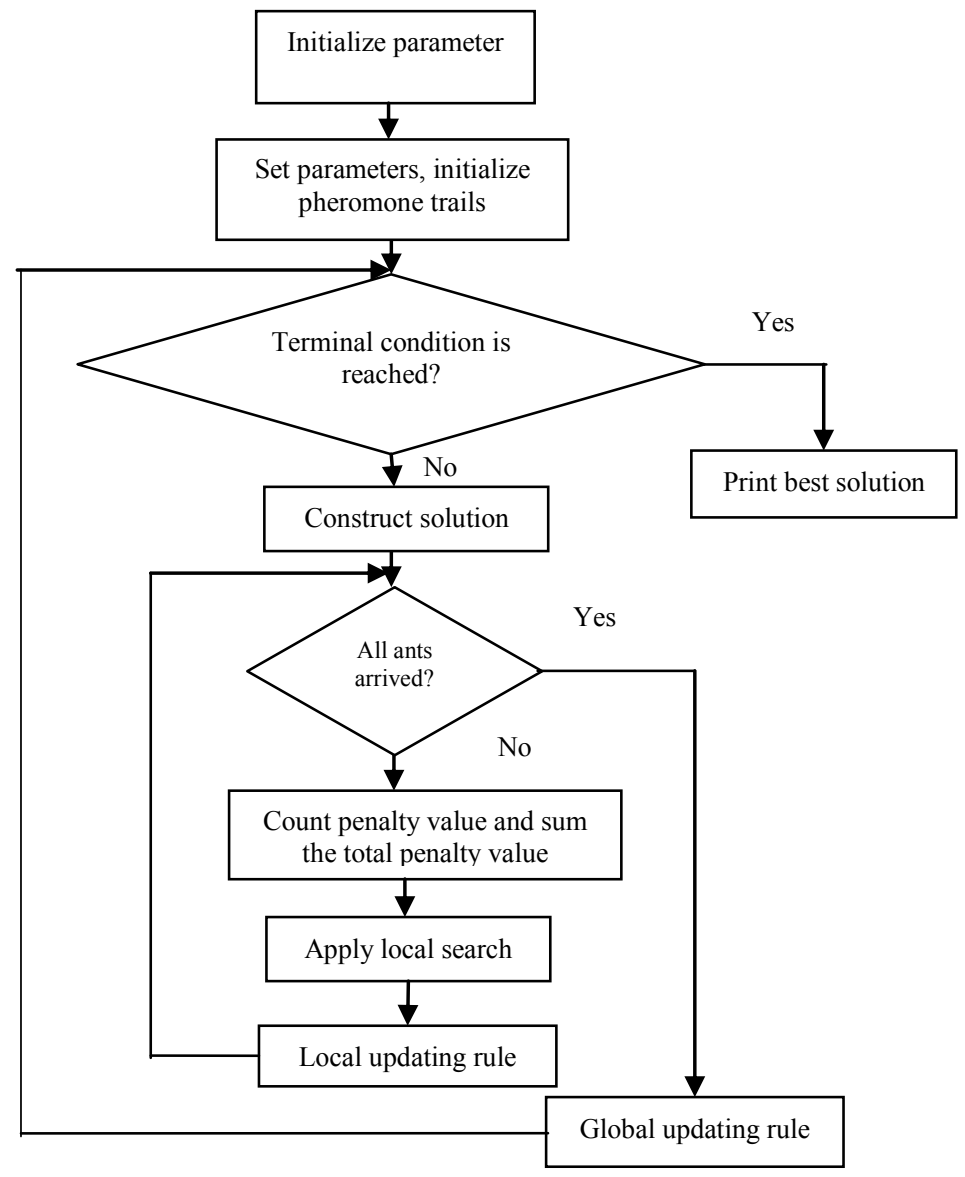

Figure 1. The flowchart of hybrid ACO algorithm as adapted from Zhang et al. (2006).

Step 1: Set the parameter of nurse scheduling. The related parameters are as follows:

- The number of staff in the roster

- The length of the roster

- $\quad$ Number of public off days 
Step 2: Set the parameter of ACO algorithm:

- $\quad$ Evaporation value of pheromone $(\rho)$

- $\quad$ Number of one group of ants $(m)$

- $\quad \alpha$ value and $\beta$ value

- $\quad$ Number of iterations

Step 3: Construct the nurse roster

- $\quad$ First, a set of $N$ shifts each is assigned based on the constraints for a year planning horizon, first for the senior level nurses and then followed by the junior level nurses in a staggered manner. The purpose of preassigned shifts is to make sure that each nurse has an equal number of night shifts that are generally disliked by nurses.

- $\quad$ After that, the shift and off days are assigned according to the constraints randomly. One group of rosters that represent one ant solution is obtained.

Step 4: Apply local search

- After the initial roster is generated, a local search algorithm known as hill climbing is applied to improve the solution quality. Particularly, Swap and Move operators are applied to the solution. The Swap operator improves the solution by exchanging between nurses, while the Move operator ejects a nurse's shift from its current position and inserts it at another position, as employed by Burke and Newall (1999), who reported good results after incorporating the hill climbing technique. Then, the performance of this group of rosters is calculated.

Step 5: Local updating of pheromone

- When each ant has finished developing a roster, the performance of each roster is compared and the pheromone is updated. If the current roster has a smaller penalty value than the previous roster, the pheromone is added to the current roster and the pheromone value from the previous one would decrease.

- While constructing its tour, an ant would modify the amount of pheromone on the visited roster by applying the local updating rule.

$$
\begin{array}{ll}
\tau(i, j) & (1-\rho) * \tau(i, j)+(\text { Constant Value / penalty of the roster }) \\
\tau(i, j) & (1-\rho) * \tau(i, j)
\end{array}
$$


where $\rho$ is the coefficient representing pheromone evaporation (note: $0<\rho<1$ ). Equation (2) is used when the ant finds a good roster so that the quantity of pheromone can be increased given by ( $Q /$ Penalty $i, j)$, where $Q$ is a constant value and penalty $i, j$ is the penalty of the roster. Next, equation (3) is used when the ant finds a bad roster from the prior roster as the amount of pheromone is reduced by a factor $(1-\rho)$. Step 4 is repeated until the whole group of ants had finished their work.

Step 6: Global updating of pheromone

- After all the ants in one group have completed their entire journey, pheromone is updated for the currently optimized roster to adhere to the global updating rule. In this step, a simple form of the rank-based mechanism is used, as introduced by Bullnheimer, Hartl, and Strauss (1999). The currently optimized roster is not necessarily available with this group of ants, as it may be with the previous group of ants.

- $\quad$ The pheromone is updated by using the following formula:

$(1-\rho)^{*} \tau(i, j)+\left((\sigma-\mu)^{*}(\right.$ Constant Value / Penalty value of the roster $)+$ $\sigma^{*}$ ( Constant Value /Penalty value of the best roster found) (4)

Where $L \mu$ is the penalty value of the roster and $L^{*}$ is the penalty value of the best roster found.

Step 7: Update the current best combination. Distinguish whether the stop condition has been met, that is, whether all the groups of ants have completed their search journey and to provide the best solution.

The ACO requires many parameters to be tested in order to obtain good solutions to any problem. Therefore, in this study, the hybrid ACO model was developed and tested with different sets of parameters for $\alpha, \beta$, and $\rho$, as suggested by Dorigo et al. (1996) in which is $\alpha=1, \beta=5, \rho=0.5$ and $Q$ $=100$ (to be known as ACO1) and Zhang et al. (2006) where $\alpha=0.1, \beta=2$, $\rho=0.01$ and $Q=.100$ (to be known as ACO2). These two previous studies were referred as their ACO techniques provided good solutions when those parameter settings were used. Hence, a similar usage of these parameter settings was relevant in the testing of our hybrid ACO for the NRP problem.

\section{RESULTS AND DISCUSSION}

The algorithm was applied and tested using real data to ascertain its effectiveness and potential. The experimentation of different sets of parameters was made to 
obtain a better performance of the algorithm in this NRP. The ACO algorithm was tested with different sizes of initial ants. The results displayed in Table 2 shows that the increment in size for the initial ants generated a higher probability of best solutions with higher pheromone values. Meanwhile, a smaller number of initial ants have resulted in lower pheromone values. The pheromone values and penalties obtained are presented in Table 2 and in Figures 2 and 3. Figure 2 shows the initial ants with pheromone values, while Figure 3 shows the initial ants with penalty values.

Table 2

Computational results with different sizes of initial ants

\begin{tabular}{c|cccccccccc}
\hline $\begin{array}{c}\text { Initial } \\
\text { ants }\end{array}$ & 10 & 20 & 30 & 40 & 50 & 60 & 70 & 80 & 90 & 100 \\
$\begin{array}{c}\text { Pheromone } \\
\text { value }\end{array}$ & 24.88 & 55.71 & 119.57 & 199.52 & 496.92 & 1183.50 & 1391.70 & 796.56 & 896.88 & 1991.70 \\
Penalty & 80 & 70 & 50 & 40 & 20 & 10 & 10 & 20 & 20 & 10 \\
\hline
\end{tabular}

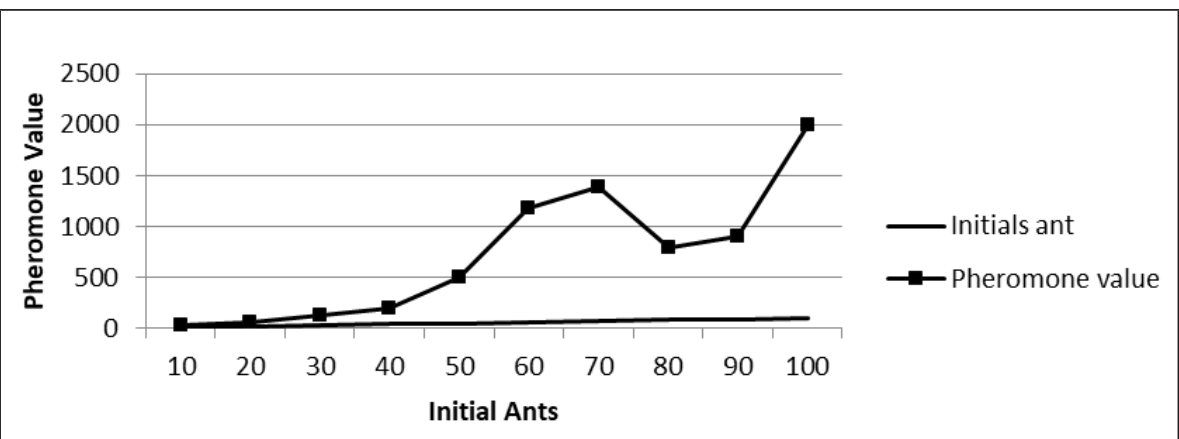

Figure 2. Initial ants with pheromone values.

Based on Figure 2, it can be concluded that the larger the group of ants, more pheromone trails are available so that other ants can use the information to be greedy, in addition to being adaptive. Besides, these ants have good working memory, which is essential to ensure that only feasible solutions are generated. Figure 3 shows that a least penalty value of 10 is obtained when the number of initial ants is 60,70 and 100 . The stopping criteria used is the number of iterations and stagnation stages by the ants. However, good rosters can be produced before entering the stagnation stage during execution time. 


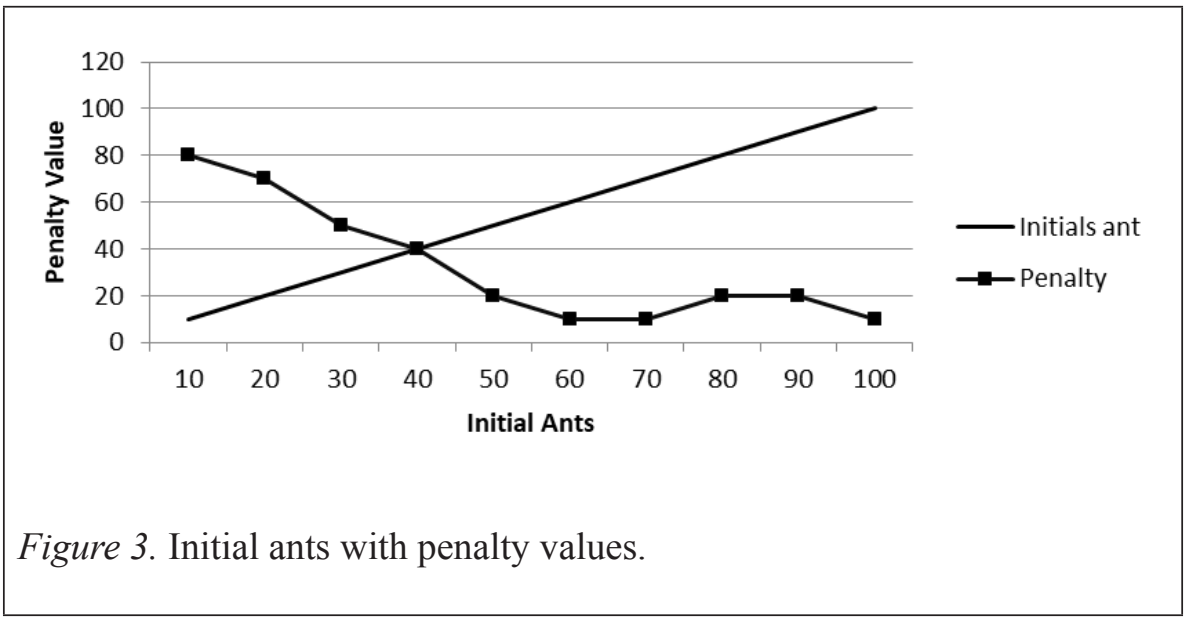

The variations of our hybrid ACO model were each run with 100 iterations, which was the same number of iterations experimented and recommended by Ramli et al. (2004). A total of 60 initial ants were used in the experiment. The results for 30 sets of sample rosters as tabulated in Table 3 were obtained by running each roster from hybrids $\mathrm{ACO} 1$ and $\mathrm{ACO} 2$ of the experiment. Experimental observations showed that $\alpha=1, \beta=5, \rho=0.5$, and $Q=100$ still appeared to be the better choice for these parameters, as suggested by Dorigo et al. (1996). The best rosters were found in samples 2 and 13 with a penalty value equivalent to 10 . On the other hand, hybrid ACO2 obtained the lowest penalty value of 20 which was evidenced in samples 11 and 30 . There was only a slight difference in the performance of the generated rosters, which could be attributed to the effective design of the proposed hybrid ACO model.

\section{Table 3}

Minimum penalty values obtained from two variations of the ACO hybrid models

\begin{tabular}{ccc}
\hline Sample of Roster & Hybrid ACO1 & Hybrid ACO2 \\
\hline 1 & 40 & 60 \\
2 & 10 & 40 \\
3 & 50 & 40 \\
4 & 30 & 40 \\
5 & 30 & 20 \\
6 & 40 & 50 \\
\hline
\end{tabular}




\begin{tabular}{|c|c|c|}
\hline Sample of Roster & Hybrid ACO1 & Hybrid ACO2 \\
\hline 7 & 20 & 30 \\
\hline 8 & 30 & 40 \\
\hline 9 & 30 & 60 \\
\hline 10 & 30 & 40 \\
\hline 11 & 30 & 20 \\
\hline 12 & 30 & 30 \\
\hline 13 & 10 & 50 \\
\hline 14 & 30 & 50 \\
\hline 16 & 40 & 40 \\
\hline 17 & 40 & 40 \\
\hline 18 & 30 & 40 \\
\hline 19 & 20 & 30 \\
\hline 20 & 50 & 30 \\
\hline 21 & 40 & 40 \\
\hline 22 & 30 & 30 \\
\hline 23 & 40 & 40 \\
\hline 24 & 20 & 40 \\
\hline 25 & 30 & 40 \\
\hline 26 & 20 & 50 \\
\hline 27 & 30 & 50 \\
\hline 28 & 40 & 40 \\
\hline 29 & 30 & 30 \\
\hline 30 & 40 & 20 \\
\hline
\end{tabular}

In addition, the performance of the proposed hybrid ACO for both sets of parameter settings were also further evaluated, qualitatively, based on four performance criteria i.e. coverage, quality, flexibility, and cost. The evaluations were conducted together with the performance of another similar nurse rostering model that used the memetic algorithm (MA) Ramli et al., 2017) for comparison purposes. In addition, the hybrid ACO model was also compared with a manually or human generated schedule that was being practised in the actual NRP at a large general hospital in Malaysia. These descriptive evaluations are presented in Table 4. Overall, the performance of the hybrid ACO and MA were comparable. 
Table 4

Comparison of ACO hybrids based on performance criteria

\begin{tabular}{|c|c|c|c|c|c|}
\hline Criteria & Measurements & $\begin{array}{l}\text { Hybrid } \\
\text { ACO1 }\end{array}$ & $\begin{array}{l}\text { Hybrid } \\
\text { ACO2 }\end{array}$ & $\begin{array}{c}\text { MA } \\
\text { Model }\end{array}$ & $\begin{array}{l}\text { Human } \\
\text { Generated }\end{array}$ \\
\hline Coverage & $\begin{array}{l}\text { Minimum } \\
\text { coverage } \\
\text { requirements } \\
\text { met. }\end{array}$ & Yes & Yes & Yes & Yes \\
\hline Quality & $\begin{array}{l}\text { - Equalization } \\
\text { of night } \\
\text { shift rotation } \\
\text { among staff. } \\
\text { - Equalization } \\
\text { of other shifts } \\
\text { distribution } \\
\text { in a particular } \\
\text { period. } \\
\text { - Violation of } \\
\text { not more than } \\
\text { six-day work } \\
\text { stretch. } \\
\text { - Violation } \\
\text { of circadian } \\
\text { ordering. } \\
\text { - Existence of } \\
\text { single-day } \\
\text { work stretch. } \\
\text { - Ability to } \\
\text { assign public } \\
\text { holidays } \\
\text { in their } \\
\text { designated } \\
\text { week or the } \\
\text { following } \\
\text { week. }\end{array}$ & 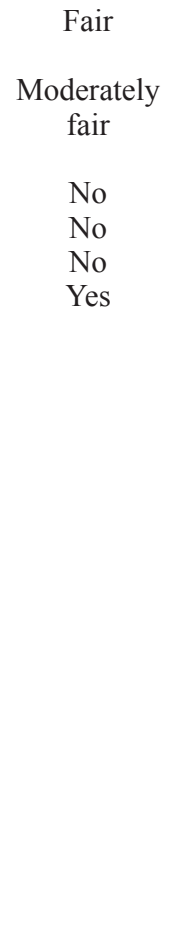 & 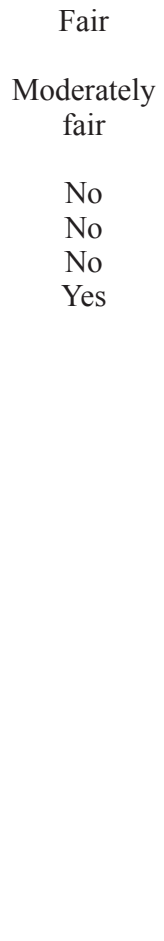 & 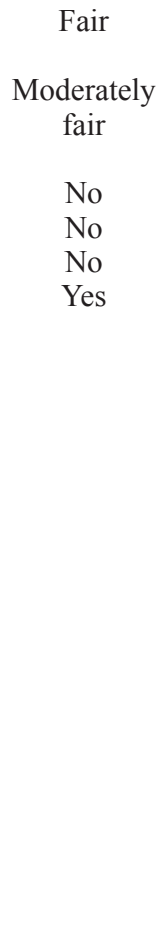 & $\begin{array}{l}\text { Fair } \\
\text { Not fair }\end{array}$ \\
\hline Flexibility & $\begin{array}{l}\text { - Adapting to } \\
\text { change at } \\
\text { staffing level. } \\
\text { - Privilege of } \\
\text { choice in } \\
\text { selecting the } \\
\text { final roster, } \\
\text { as desired. }\end{array}$ & $\begin{array}{l}\text { Easy } \\
\text { Yes }\end{array}$ & $\begin{array}{l}\text { Easy } \\
\text { Yes }\end{array}$ & $\begin{array}{l}\text { Easy } \\
\text { Yes }\end{array}$ & $\begin{array}{c}\text { Moderately } \\
\text { difficult } \\
\text { No }\end{array}$ \\
\hline
\end{tabular}




\begin{tabular}{|c|c|c|c|c|c|}
\hline Criteria & Measurements & $\begin{array}{l}\text { Hybrid } \\
\text { ACO1 }\end{array}$ & $\begin{array}{l}\text { Hybrid } \\
\text { ACO2 }\end{array}$ & $\begin{array}{c}\text { MA } \\
\text { Model }\end{array}$ & $\begin{array}{l}\text { Human } \\
\text { Generated }\end{array}$ \\
\hline Cost & $\begin{array}{l}\text { - Time } \\
\text { required in } \\
\text { constructing } \\
\text { a roster. } \\
\text { - Category of } \\
\text { staff required } \\
\text { to construct a } \\
\text { roster. }\end{array}$ & $\begin{array}{c}\text { About } 1 \\
\text { minute } \\
\text { Any senior } \\
\text { staff }\end{array}$ & $\begin{array}{l}\text { About } 1 \\
\text { minute } \\
\text { Any senior } \\
\text { staff }\end{array}$ & $\begin{array}{l}\text { About } 12 \\
\text { Minutes } \\
\text { Any senior } \\
\text { staff }\end{array}$ & $\begin{array}{l}\text { 3-8 hours } \\
\text { Head nurse/ } \\
\text { Assistant }\end{array}$ \\
\hline
\end{tabular}

Based on the four criteria mentioned, the rosters generated from our Hybrid ACO1 and ACO2 demonstrated good and fair performance in terms of satisfying all the hard and soft constraints of the NRP. Hence, the proposed rosters were of high quality. Both variations of the hybrid ACO provided very good rosters with only minor violations of soft constraints, where the penalty value for the best-so-far roster generated by hybrid ACO1 was only 10 , while that of hybrid ACO2 was 20 . As shown in Table 3, it was found that only one soft constraint was violated with hybrid ACO1. This violated soft constraint was regarding the arrangement of $\mathrm{E}$ shifts before the $\mathrm{M}$ shifts, which was generally disliked by nurses. Similarly, the same soft constraint was violated twice with hybrid ACO2. This comparison is also summarized in Table 5. On the other hand, there were issues in terms of quality, flexibility and cost for the human generated roster. Therefore, with reference to the criteria, both parameter settings exhibited good performance for the hybrid ACO. However, for a detailed comparison, the difference in penalty values is the final yardstick. Thus, hybrid ACO1 which adopted the parameter settings suggested by Dorigo et al. (1996) with $\alpha=1, \beta=5, \rho=0.5$ seemed a better alternative for solving the NRP. The performance of ACO in addressing NRPs still depends on the parameter values and the number of ants. Hence, more experimentation is recommended in finding the best solution without any penalty value.

Both the hybrid ACO models were also compared in detail with the MA model, i.e., the Memetic Algorithm for the same NRP. The comparison results based on the penalty value and time taken are shown in Table 5. The results indicated that the hybrid ACO was a more effective technique to address the NRP. All hard constraints were fulfilled with minimum violations in the soft constraints. It shows that the hybrid ACO technique is able to satisfy almost all the nurses' preferences. 
Table 5

Comparison in terms of penalty values and time taken using the metaheuristic technique

\begin{tabular}{lcc}
\hline \multicolumn{1}{c}{ Metaheuristic Technique } & Best Penalty Value & $\begin{array}{c}\text { Time Taken } \\
\text { (in seconds) }\end{array}$ \\
\hline Hybrid ACO 1 & 10 & 29.55 \\
Hybrid ACO 2 & 20 & 29.55 \\
MA & 2397 & 621.10 \\
\hline
\end{tabular}

\section{CONCLUSION AND FUTURE WORK}

Efficient rosters are needed to fulfill hard hospital constraints and soft constraints based on nurses' preferences. Thus, in relation to our study, the objective of generating efficient rosters goes beyond satisfying nurses' preferences, especially the allocation of consecutive off days, while ensuring that sufficient nurses are on duty for every shift on a daily basis. All nurses' work shifts can be assigned effectively and the desired off days can be attained to enable them to get adequate rest before continuing work the next day. This could eventually increase the job satisfaction of nurses. However, certain aspects of rostering can be explored in future by considering the capability of re-rostering in a particular rostering model. Hence, the issue of real time or ad-hoc allocation of nurses especially in cases of emergency absences could be overcome.

\section{ACKNOWLEDGMENT}

This research received no specific grant from any funding agency in the public, commercial, or non-profit sectors.

\section{REFERENCES}

Abreu, N., Ajmal, M., Kokkinogenis Z., \& Bozorg B. (2011). Ant Colony Optimization. Technical Report, University of Porto, Portugal.

Aknine, S., Pinson, S., \& Shakun, M. F. (2004). An extended multi-agent negotiation protocol, Autonomous Agents and Multi-Agent Systems, $8(1), 5-45$. 
Aickelin, U., \& Dowsland, K. A. (2000). Exploiting problem structure in a genetic algorithm approach to a nurse rostering problem. Journal of Scheduling, 3(3), 139-153.

Aickelin, U., \& Dowsland, K. A. (2003). An indirect genetic algorithm for a nurse scheduling problem. Computers \& Operations Research, 31(5), 761-778.

Awadallah, M. A., Al-Betar, M. A., Khader, A. T., Bolaji, A. L., \& Alkoffash, M. (2017). Hybridization of harmony search with hill climbing for highly constrained nurse rostering problem. Neural Computing and Applications, 28(3), 463-482.

Awadallah, M. A., Bolaji, A. L., \& Al-Betar, M. A. (2015). A hybrid artificial bee colony for a nurse rostering problem. Applied Soft Computing, 35, 726-739.

Arajy, Y. Z., Abdullah, S., \& Kifah, S. (2017). Non-liner great deluge algorithm for handling nurse rostering problem. International Journal of Applied Engineering Research, 12(15), 4959-4966.

Azaiez, M. N., \& Al Sharif, S. S. (2005). A 0-1 goal programming model for nurse scheduling. Computers \& Operations Research, 32(3), 491-507.

Baumelt, Z. et al. (2007). Hospital Nurse Scheduling (Unpublished Master Thesis). Department of Control Engineering, Faculty of Electrical Engineering, Czech Technical University in Prague.

Bester, M. J., Nieuwoudt, I., \& Vuuren, J. H. V. (2007). Finding good nurse duty schedules: A case study. Journal of scheduling, 10(6), 387-405.

Bullnheimer, B, Hartl, R. F., \& Strauss, C. (1997). A new rank-based version of the ant system: A computational study (Working Paper No.1). Retrieved from http://epub.wu.ac.at/english/wp

Burke, E. K., Curtois, T., Qu, R., \& Berghe, G. V. (2007). A scatter search for the nurse rostering problem. Computer science technical report. University of Nottingham.

Cheang, B., Li, H., \& Rodrigues, B. (2003) Nurse rostering problems - a bibliographic survey. European Journal of Operational Research, 151, 447-460.

Chiaramonte, M. V., \& Chiaramonte, L. M. (2008). An agent-based nurse rostering system under minimal staffing conditions. International Journal of Production Economics. 114(2), 697-713. doi:10.1016/j. ijpe.2008.03.004.

Chiaramonte, M. V. (2008). Competitive nurse rostering and rerostering (Unpublished Ph.D. Thesis). Arizona State University.

Croce, F. D., \& Salassa, F. (2014). A variable neighborhood search based matheuristic for nurse rostering problems. Annals of Operations Research, 218(1), 185-199. 
Dorigo, M., Maniezzo, V., \& Colorni, A. (1996). The ant system: Optimization by a colony of cooperating agents. IEEE Transactions on System, Man, and Cybernatics-Part B, 26(1), 29-41

Dowsland, K. (1998). Nurse scheduling with tabu search and strategic oscillation. European Journal of Operational Research, 106, 393-407.

Dowsland, K., \& Thompson, J. (2000). Solving a Nurse Scheduling Problem with Knapsacks, Networks and Tabu Search. The Journal of the Operational Research Society, 51(7), 825-833.

Eskandari, A., \& Ziarati, K. (2008). Nurse rostering using fuzzy logic: A case study. Journal of Industrial Engineering International, 4(7), 69-82.

Fumiko, Y. (2014). Implications of circadian rhythm disruptions for nurse managers. Saku University Journal of Nursing, 6(1), 65-74.

Gonsalves, T., \& KoheiKuwata, (2015). Memetic algorithm for the nurse scheduling problem, International Journal of Artificial Intelligence \& Applications (IJAIA), 6(4), 43-52.

Gutjahr, W. J., \& Rauner, M. S. (2007). An ACO algorithm for a dynamic regional nurse scheduling problem in Austria. Computers \& Operations Research, 34(3), 642-666.

Haspeslagh, S., Causmaecker, P. D., \& Berghe G. V. (2007). Distributed Decision Making in Hospital Wide Nurse Rostering Problems. Proceedings of the Multidisciplinary International Scheduling Conference, 28-31 August 2007, Paris.

Hayakawa, D., Mizuno, K., Sasaki, H., \& Nishihara, S. (2011). Improving Search Efficiency Adopting Hill-Climbing to Ant Colony Optimization for Constraint Satisfaction Problems. 2011 Third International Conference on Knowledge and Systems Engineering, 14-17 October 2011, Hanoi.

Ibrahim, A. O., Shamsuddin, S. M., \& Qasem, S. N. (2015). Hybrid NSGA-II Optimization for Improving the Three-Term Bp Network for Multiclass Classification Problems. Journal of Information and Communication Technology, 14, 21-38.

Joseph, M. L. (2018). Staffing and Scheduling. In L. D. Huber (Eds.), Leadership and nursing care management (5th ed.). Missouri, US: Elsevier.

Kundu, S., \& Acharyya, S. (2008). A SAT approach for solving the nurse scheduling problem. Proceedings of the 2008 IEEE Region 10 Conference. 19-21 November 2008, Hyderabad, India.

Kundu, S., Mahato, M., Mahanty, B., \& Acharyya, S. (2008). Comparative performance of simulated annealing and genetic algorithm in solving nurse scheduling problem. Proceedings of the International MultiConference of Engineers and Computer Scientists. Vol I IMECS 2008, 19-21 March 2008, Hong Kong. 
Lim, A., Lin, J., Rodrigues, B., \& Xiao. F. (2006). Ant colony optimization with hill climbing for the bandwidth minimization problem. Applied Soft Computing, 6(2), 180-188.

Liu, Z., Liu, Z., Zhu, Z., Shen, Y., \& Dong, J. (2018). Simulated annealing for a multi-level nurse rostering problem in hemodialysis service. Applied Soft Computing, 64, 148-160.

Moz, M., \& Pato, M. V. (2007). A genetic algorithm approach to a nurse rerostering problem. Computers \& Operations Research, 34(3), 667691.

Oonsrikaw, Y., \& Thammano, A. (2018). Enhanced Ant Colony Optimization with Local Search. IEEE/ACIS 17th International Conference on Computer and Information Science (ICIS). 24-26 May 2017, Wuhan.

Osman, I. H. (1993). Metastrategy simulated annealing and tabu search algorithms for the vehicle routing problem. Annals of Operations Research, 41, 421-451.

Özcan, E. (2005). Memetic Algorithms for Nurse Rostering. Technical report. Istanbul, Turkey: Yeditepe University.

Pooja, L., \& Rupali, M. N. (2014). Solving Nurse Rostering Problem Using Ant Colony Optimization Approach. International Journal of Modern Trends in Engineering and Research, 2(3), 162-170.

Ramli, R., Mustafa, A., \& Khader, A. T. (2004). Adopting AHP in evaluating nurse scheduling methods. Jurnal STATISTIKA: Forum Teori dan Aplikasi Statistika, 4(2), 155-160.

Ramli, R., \& Lim, H. T. (2017). Maximax \& Maximin and 2FBlockwise Operators: Enhancement in the evolutionary algorithm for a nurse scheduling problem. Journal of Telecommunication, Electronic and Computer Engineering, 9(1-2), 1-6.

Ratanavilisagul, C., \& Pasaya, B. (2018). Modified Ant Colony Optimization with Updating Pheromone by Leader and Re-Initialization Pheromone for Travelling Salesman Problem. International Conference on Engineering, Applied Sciences, and Technology (ICEAST). 4-7 July 2018, Phuket.

Rohim, N. (2013). Solving a nurse rostering problem using ant colony optimization algorithm (Unpublished Master Thesis). Universiti Utara Malaysia.

Royal College of Physicians. (2018). Work and wellbeing in the NHS: Why staff health matters to patient care. Technical Report. Royal College of Physicians. Retrieved from https://www.rcpsych.ac.uk/pdf/RCP-\%20 WorkWellbeingNHS.pdf

Shehab, M., Khader, A. T., \& Laouchedi, M. (2018). A hybrid method based on cuckoo search algorithm for global optimization problems. Journal of Information and Communication Technology, 17(3), 469-491. 
Trilling, L., Guinet, A., \& Magny, L. D. (2006). Nurse scheduling using integer linear programming and constraint programming. 12th IFAC Symposium on Information Control Problems in Manufacturing INCOM 2006. Saint-Etienne, France, Elsevier, 3. 651-656.

V'aclav'1k, R., S ucha, P., \& Hanz'alek `, Z. (2016). Roster evaluation based on classifiers for the nurse rostering problem. Journal of Heuristics, 22(5), 667-697.

Winstanley, G. (2004). Distributed and devolved work allocating planning. Applied Artificial Intelligence. 18, 97-115.

Wu, J-J., Lin, Y., \& Chen, J-y. (2013). An Ant Colony Optimization Approach For Nurse Rostering Problem. IEEE International Conference on Systems, Man, and Cybernetics. 13-16 October 2013, Manchester.

Yousefikhoshbakht, M., Didehvar, F., \& Rahmati, F. (2013). Modification of the ant colony optimization for solving the multiple traveling salesman problem. Romanian Journal of Information Science and Technology, 16(1), 65-80.

Zhang, J., Xiomin, H., Tan, X., Zhong, H., \& Huang, Q. (2006). Implementation of an ant colony optimization technique for job scheduling problem. Transaction of the Institute of Measurement and Control, 28(1), 93108. 